CERN-TH.6523/92

BI-TP 92/08

\title{
THERMAL HADRON PRODUCTION IN HIGH ENERGY HEAVY ION COLLISIONS
}

\author{
J. Cleymans ${ }^{1,2}$ and H. Satz ${ }^{2,3}$
}

\begin{abstract}
We provide a method to test if hadrons produced in high energy heavy ion collisions were emitted at freeze-out from an equilibrium hadron gas. Our considerations are based on an ideal gas at fixed temperature $T_{f}$, baryon number density $n_{B}$, and vanishing total strangeness. The constituents of this gas are all hadron resonances up to a mass of 2 $\mathrm{GeV}$; they are taken to decay according to the experimentally observed branching ratios. The ratios of the various resulting hadron production rates are tabulated as functions of $T_{f}$ and $n_{B}$. These tables can be used for the equilibration analysis of any heavy ion data; we illustrate this for some specific cases.
\end{abstract}

1 Department of Physics, University of Cape Town, Rondebosch 7700, South Africa

2 Fakultät für Physik, Universität Bielefeld, D-4800 Bielefeld 1, Germany

3 Theory Division, CERN, CH-1211 Geneva 23, Switzerland

CERN-TH.6523/92

BI-TP $92 / 08$

May 1992 


\section{Introduction}

Five years ago, an extensive program for the study of strongly interacting matter through high energy heavy ion collisions was initiated at CERN and BNL [1-5] A crucial element in this endeavour is the production of systems which are indeed thermalized and not just an uncorrelated superposition of nucleon-nucleon collisions. Thermalisation can only be achieved through sufficient rescattering of the incoming nucleons among each other and with secondaries, and of the secondaries among each other. It is not obvious if, how and at what stage thermalisation is achieved. If it is achieved at all, and if it is not destroyed subsequently by possible non-equilibrium features [6-11], then the produced hadrons should be observed in ratios determined by the thermodynamics of a system at freeze-out temperature $T_{f}$ and freeze-out baryon density $n_{B}$. The aim of this work is to determine and tabulate these ratios for the various hadron species as function of $T_{f}$ and $n_{B}$. Our tables should allow a thermalisation analysis of any present and future heavy ion data, and we shall illustrate how such an analysis is carried out. We should emphasize that we are not providing a model of any kind for the space-time development of the systems produced, but merely a check of the degree of thermalisation indicated in the observed hadron ratios. In particular, the collisions between light ion projectiles and heavy ion targets studied studied up to now very likely do not lead to complete thermalisation, and so we expect experiments to deviate from the ratios listed here. These deviations should, however, lead to values between the ratios from p-p collisions and the thermal ones. If they don't, this could be a hint for some new effect. Furthermore, if there is thermalisation, one cannot say anything about how it was established. Thermal systems have no memory, and hence the observation of thermal hadron production ratios neither excludes nor confirms quark deconfinement at an earlier stage.

It should be noted that the thermalisation we are considering here refers to the approach to a statistical equilibrium among the different hadron species. It is conceivable [11] that such a "chemical" equilibrium sets in later than a "thermal" equilibrium among different hadrons of the same species. So far, however, there seem to be no clear-cut tests for the latter.

\section{Thermal Hadron Production}

Hadronic matter in equilibrium is specified by three parameters : the temperature $T_{f}$, the baryon number density $n_{B}$, and the strangeness density $n_{S}$; the latter is zero unless one is interested in globs of strange matter [10]. Hadronic matter in thermal and chemical equilibrium will have a certain composition of particle species, characterized by "chemical" potentials. In addition to the baryon number chemical potential $\mu_{B}$, we have a strangeness chemical potential $\mu_{S}$, since particles of a given strangeness can have different baryon numbers (e.g., $\bar{K}^{0}$ and $\Lambda$ ). The value of $\mu_{B}$ is fixed by giving the overall baryon number density $n_{B}$, and that of $\mu_{S}$ by fixing the overall strangeness, 
which in our case will be zero. The number densities for all hadron states are then given in terms of only $\mu_{B}$ and $T_{f}$. There are many particle species, and if they are produced in equilibrium, all their ratios must be fixed in terms of these two parameters. It is thus straightforward to test if there was equilibrium, and since this question is so basic, it should be one of the first tests to be made.

We include only the essential interaction features of a multi-hadron system near freeze-out: resonance production in general and particle repulsion at high baryon density. The bulk of meson production is known to take place through intermediate resonance states, and we therefore start with an ideal gas containing the observed (strange and non-strange) mesonic and baryonic resonances. A crucial question here is how many of the observed resonances we should include. There is no clear-cut answer by including more and more resonance states, we evidently go back further and further in the evolution of the system. To obtain a definite scheme, we shall include all resonances up to a mass of $2 \mathrm{GeV}$. In order to test the importance of high mass contributions, we have also carried out calculations including only the resonances of the basic multiplets, i.e., the pseudoscalar meson octet plus singlet, the vector meson octet plus singlet, the baryon octet and the baryon decuplet. - To obtain the distributions of the actually observed hadrons, in particular pions and kaons, we then let the resonances decay according to the measured branching ratios. This procedure is well known; see e.g. [12].

For small or vanishing baryon number, this would suffice to fix our model; at high $n_{B}$, however, a strong, short-range repulsion sets in between baryons, even if they have different quantum numbers; this can be described in terms of excluded volumes, repulsive interaction contributions, or Pauli blocking. We shall here adopt a hardsphere picture [13], in which the baryons in the system effectively remove a portion of the available spatial volume. As a consequence, the baryon number density $n_{B}$ becomes

$$
n_{B}=\frac{n_{B}^{o}}{\left[1+V_{0} n_{B}^{0}\right]} \text {, }
$$

where $n_{B}^{o}$ denotes the density calculated for an ideal gas of pointlike baryons. We note that for $n_{B}^{o} \rightarrow \infty, n_{B} \rightarrow 1 / V$, i.e., we have dense packing* of hard-sphere baryons with an intrinsic volume $V$. In the case of several baryon species $\alpha=1,2, \ldots, r$, eq.(1) becomes

$$
n_{B}=\frac{n_{B}^{o}}{\left[1+\sum_{\alpha=1}^{r} V_{\alpha} n_{\alpha}^{0}\right]}
$$

where the baryon volume $V_{\alpha}$ could be proportional to the mass, as suggested e.g. by the bag model. Here we take one universal volume $V=4 \pi R^{3} / 3$, with $R=0.8 \mathrm{fm}$ as an indicative radius for the nucleon, and apply the excluded volume correction (1) to all particle species, mesons as well as baryons. Note that this does not imply repulsion

* Actually $1 / V$ is the limiting value for incompressible but deformable baryons; a true hard-sphere system yields $1 / c V$, with $c \geq 1[14]$. 
between mesons; it only reduces space accessible to them, because some is already "used up" by the hard-core baryons. For matter at $n_{B}=0$, the full volume is once more available; for $T=0$, we recover the cold nucleon gas with hard-sphere repulsion [13]. The correction term moreover cancels out in all particle ratios. - This completes our picture.

To illustrate the resulting pattern, we note that

$$
n_{K^{ \pm}}^{0}=\int \frac{d^{3} p}{(2 \pi)^{3}} \frac{1}{\left[e^{\left(E_{K} \mp \mu_{S}\right) / T}-1\right]}
$$

gives the number density of directly produced thermal kaons, and

$$
n_{\Lambda}^{0}=\int \frac{d^{3} p}{(2 \pi)^{3}} \frac{1}{\left[e^{\left(E_{\Lambda}-\left(\mu_{B}-\mu_{S}\right)\right) / T}+1\right]}
$$

that of directly produced thermal, pointlike $\Lambda$ 's; here $E_{K}=\left(\mathbf{p}^{2}+m_{K}^{2}\right)^{1 / 2}$ and $E_{\Lambda}=$ $\left(\mathbf{p}^{2}+m_{\Lambda}^{2}\right)^{1 / 2}$. The actual production rates are obtained from this by including baryon repulsion according to eq.(2) and by adding to eqs.(3) and (4) the corresponding values from resonance production, such as $K^{0 *} \rightarrow K^{+}+\pi^{-}$, using the experimentally determined branching ratios.

Requiring the difference between all strange particle number densities to vanish,

$$
\sum_{i} n_{S}^{i}-\sum_{i} n_{\bar{S}}^{i}=0
$$

fixes $\mu_{S}$ and thus gives us all particle densities in terms of $T$ and $\mu_{B}$. Summing at fixed $\mu_{B}$ over all baryon contributions,

$$
\sum_{i} n_{B}^{i}-\sum_{i} n_{\bar{B}}^{i}=n_{B}
$$

determines the overall baryon number density $n_{B}$. Note that in eq.(6) we sum over the physical baryon densities as defined by eq.(2). Eqs.(5) and (6) provide a relation between the strange chemical potential and the baryon chemical potential; it is shown in Fig.1. With increasing temperature, $\mu_{S}$ is seen to increase faster with $\mu_{B}$, and for temperatures $T \gtrsim 200 \mathrm{MeV}, \mu_{S} / \mu_{B}$ is found to be rather constant in the range considered here. In particular we note that for $T \simeq 200 \mathrm{MeV}$ and up to about $\mu_{B} \simeq 500 \mathrm{MeV}$, we have $\mu_{S} \simeq(1 / 3) \mu_{S}$. In terms of the baryon number and strangeness chemical potentials for quarks, $\mu_{b}$ and $\mu_{s}$, respectively, the hadronic chemical potentials become $\mu_{B}=3 \mu_{b}$ and $\mu_{S}=\mu_{b}-\mu_{s}$. Hence $\mu_{B} \simeq 3 \mu_{S}$ just translates into $\mu_{s} \simeq 0$. Since this arises here quite naturally in a hadronic picture, it is difficult to consider it as evidence for a primordial quark-gluon plasma [15].

In Fig. 2 we illustrate the effect of hard-core repulsion between baryons, by plotting $n_{B}$ as function of $\mu_{B}$ for a hard-core volume $V=0$ and for one with radius $R=0.8$ 
fm. We note that the repulsion begins to become crucial around $n_{B} \simeq 0.1 \mathrm{fm}^{-3}$, i.e., already below standard nuclear density .

We should emphasize that the particle ratios which we will discuss in this paper are by construction independent of the specific relation between the baryon chemical potential and the baryon density: as already mentioned, the repulsion introduced in equation (1) cancels out in the ratios. The relation between baryon number density and baryon chemical potential, on the other hand, does depend on the specific form (1). In the tables given at the end of this paper, the baryon number density is determined by this form with the hard core volume as defined there.

\section{Thermal Hadron Rates}

In this section we will discuss the general features of the various hadron species. Since we are here only interested in the qualitative behaviour, the results for this section were obtained using only the basic hadron multiplets, unless otherwise noted.

\subsection{Pions}

Pions are present in thermal equilibrium in the heat bath and in addition in the decay products of almost all heavier particles. As long as the temperature is below 100 $\mathrm{MeV}$, only very few heavy particles are present and almost all pions are of direct thermal origin. It is well known, however, that the number of directly produced thermal pions decreases at higher temperatures (see e.g. [6,7],[12],[16-19]). This becomes evident in Fig.3, where the ratio of all pions (i.e., including decay products) to direct thermal pions is plotted as a function of temperature. It increases rapidly above $100 \mathrm{MeV}$. For higher temperatures, the total positive pion density is thus given by

$$
\begin{aligned}
n_{\pi^{+}} & =n_{\pi^{+}}(\text {thermal }) \\
& +0.285 n_{\eta} \\
& +\frac{2}{3} n_{\rho}+n_{\omega}+0.134 n_{\phi} \\
& +\frac{1}{3} n_{K^{*}}+\frac{1}{3} n_{\bar{K}^{*}} \\
& +\frac{1}{3} n_{\Delta}+\frac{1}{3} n_{\Sigma^{*}}+\frac{1}{3} n_{\Xi^{*}} \\
& +\frac{1}{3} n_{\bar{\Delta}}+\frac{1}{3} n_{\bar{\Sigma}^{*}}+\frac{1}{3} n_{\bar{\Xi}^{*}} \\
& +\cdots,
\end{aligned}
$$

in which the different factors in front of the resonance densities are obtained from the corresponding branching ratios into positive pions. As the temperature is increased still further, more and more terms will start contributing in the sum in equation (7). At fixed temperature, the ratio of all pions to thermal pions furthermore increases rapidly as a function of baryon chemical potential. This is shown in Fig.4 for two 
different values of the temperature $T$. The increase is due to the fact that the number of baryonic resonances increases with the baryon chemical potential, and these provide more pions as decay products. We notice that the fraction of thermal pions quickly becomes very small; at high temperatures and densities, it is negligible compared to the number of pions from decay products of heavier resonances. It is therefore important to specify how many such resonances are taken into account in the hadron gas, since the number of pions keeps increasing as more and more resonances are included. This dependence has been investigated and was found to be important $[12,16,20]$. In principle one must include all resonances; in practice, the contributions from heavy states become important only when the temperature of the system is relatively high. We show in Fig.3 both the result obtained from our tables, which include all observed resonances up to a mass of $2 \mathrm{GeV}$, and the same ratio obtained by including only the fundamental hadron multiplets. We see that up to temperatures of about $150 \mathrm{MeV}$, including more heavy resonances has essentially no effect; at $200 \mathrm{MeV}$, however, the ratio of all to thermal pions has already increased by about 50\%. In any case, most pions in the final state of a relativistic heavy ion collision are certainly decay products of resonances. The pion spectra therefore do tell us something about equilibrium, but only in a very indirect way.

\subsection{Kaons}

Kaons are at first sight similar to pions. We see in Fig.5 that the ratio between the total number of kaons and those that are solely of thermal origin also rises rapidly above a temperature of $100 \mathrm{MeV}$, just as is the case with pions, even though the rise is not nearly as steep. Decay kaons come mainly from strange mesonic resonances; baryons only rarely have kaons in their decay products. Even strange baryons do not decay copiously into kaons, witness the baryons in the decuplet, where only the $\Omega^{-}$ gives kaons. As function of the baryon number density, the ratio of all kaons to thermal kaons therefore does not behave like the corresponding ratio for pions; it decreases with increasing $\mu_{B}$, as seen in Fig.6.

The total density of positive kaons is determined by the relation

$$
n_{K^{+}}=n_{K^{+}}(\text {thermal })+0.495 n_{\phi}+0.5 n_{K^{*}}+\cdots .
$$

When the temperature is increased much beyond $150 \mathrm{MeV}$, more terms would have to be added to the right-hand side of the above equation. As mentioned, the baryons give almost no contributions, in contrast to the pion case (7), where they contribute very much. Nevertheless, we conclude again that a considerable part of the kaons in the final state of a relativistic heavy ion collision are not of direct thermal origin, but decay products of strange mesonic resonances. The situation here is somewhat less pronounced than it was for pions, however, where those of direct thermal origin could almost be neglected. The tables at the end of this paper were calculated using equation (8) without any further resonance contributions. 


\subsection{Vector Mesons}

Vector mesons have attracted much attention, with the observation of $J / \psi$ suppression compared to the Drell-Yan continuum and of the enhancement of $\phi$ production compared to that of $\rho$ and $\omega[5] . J / \psi$ production lies outside present considerations, since the $J / \psi$ is too heavy to be produced by thermal collisions. We therefore turn our attention to the ratio $\phi /(\rho+\omega)$, which is shown in Fig.7 as a function of temperature. This ratio is independent of the baryon chemical potential, since non-strange mesons are not sensitive to the baryonic content of the hadronic gas. Strange mesons are sensitive to $n_{B}$ through the strange chemical potential, e.g. via associated production of $\Lambda$ and $K^{+}$. The $\phi$-meson is of particular interest, because its quark structure never enters the hadronic gas calculations; from a hadronic point of view, the $\phi$ is just a heavy $\omega$. Only on the quark level does the $s \bar{s}$-content enter. - With increasing $\mu_{B}$, the $\phi /(\rho+\omega)$ ratio actually drops, because the relative weight of the $\phi$ decreases. as shown in Fig.8.

The order of magnitude of the ratio $\phi /(\rho+\omega)$ is given by

$$
\phi /(\rho+\omega) \sim \frac{1}{2} \exp \left[\left(m_{\rho}-m_{\phi}\right) / T\right]
$$

For $T=200 \mathrm{MeV}$, this gives a value of 0.14 ; the actual distributions lead to slightly larger values than the estimate (9). We have not taken into account vector mesons originating from the decay of heavy resonances, since the relevant branching ratios are not very well established. Their inclusion would lower the ratio, since it will mainly enhance the number of $\rho$-mesons.

\subsection{Strange Baryons}

Since it is experimentally difficult to separate $\Sigma^{0}$ and $\Lambda$, we shall consider these together and denote by $n_{\lambda}$ the overall production rate of the two species. Again there are non-negligible decay contributions, from the decuplet baryons $\Sigma^{*}(1.385)$ and $\Omega^{-}$ (1.672), which decay into $\Lambda$ 's and $\Sigma$ 's. We therefore get

$$
n_{\lambda}=n_{\Lambda}(\text { thermal })+\frac{1}{3} n_{\Sigma}(\text { thermal })+0.9 n_{\Sigma^{*}}+0.68 n_{\Omega}+\cdots,
$$

where $n_{\Sigma}$ counts all charge states, so that $n_{\Sigma} / 3$ gives us the neutrals. - In a similar way, $\Xi$ gets contributions from its decuplet partner the $\Xi^{*}(1.530)$, leading to

$$
n_{\Xi}=n_{\Xi}(\text { thermal })+n_{\Xi^{*}}+\cdots .
$$

In Fig.9 we show the relative importance of the $\Xi(1.32 \mathrm{GeV})$ in comparison to the $\Xi^{*}$ $(1.53 \mathrm{GeV})$, as a function of temperature for a fixed $\mu_{B}=200 \mathrm{MeV}$. Here we plot the ratio $\left(\Xi+\Xi^{*}\right) / \Xi$, i.e., (all $\Xi /$ thermal $\Xi$ ). Again one observes a quick increase of this ratio above temperatures of $100 \mathrm{MeV}$, similar to the one observed in Fig.5 for kaons. This shows that even a heavy particle like the $\Xi$ is strongly influenced by heavier resonances.

There thus seems to be no case where heavy resonances can be neglected. Contrary to 
the kaon case, the ratio considered here is independent of the baryon chemical potential, because both particles are baryons with identical quantum numbers and all dependence on chemical potentials therefore cancels out. The tables at the end of this paper were calculated using all resonances having masses up to about $2 \mathrm{GeV}$.

To obtain an order of magnitude estimate of the $\bar{\Lambda} / \Lambda$ ratio, one can use

$$
(\bar{\Lambda} / \Lambda) \sim \exp \left[\left(-2 \mu_{B}+2 \mu_{S}\right) / T\right],
$$

while for the $\bar{\Xi} / \Xi$ ratio one has

$$
(\bar{\Xi} / \Xi) \sim \exp \left[\left(-2 \mu_{B}+4 \mu_{S}\right) / T\right] .
$$

These estimates neglect the dependence on transverse momentum; in this case, the $\Lambda$ and the $\Lambda^{*}$ (and similarly the $\Xi$ and the $\Xi^{*}$, as well as the corresponding antibaryons) have the same dependence on $\mu_{B}$. Hence all "all/thermal" ratios are here approximately $\mu_{B}$-independent. However, since the momenta were neglected, these results should be used only as rough approximations. Nevertheless, the inequality

$$
(\bar{\Xi} / \Xi)>(\bar{\Lambda} / \Lambda)
$$

will hold even for the complete calculation, since the ratio (12) contains a factor $2 \mu_{S}$ less in the exponent than the ratio (13).

\section{The Analysis of Equilibrium Behaviour}

We will now illustrate for some specific cases how our tables can be used to study the degree of equilibration found in heavy ion data, and what conclusions one might draw from the results.

\subsection{Strange Baryons}

Here we shall consider the data on strange baryons provided by the WA85 [2] and NA35 [3] collaborations at CERN. From two measured ratios we can estimate the two thermal parameters, i.e. the temperature and the baryon chemical potential. The data of [2] provide the ratios $\bar{\Lambda} / \Lambda=0.13 \pm 0.03$ and $\Xi / \Xi=0.39 \pm 0.07$. Each leads to a "band" in the $T_{f}-\mu_{B}$ plane, which can be determined from our tables. The result is shown in Fig.10, where we see that the two bands cross in the rather small shaded region. From this we would get the ranges $170 \mathrm{MeV} \leq T_{f} \leq 220 \mathrm{MeV}$ for the freezeout temperature and $220 \mathrm{MeV} \leq \mu_{B} \leq 420 \mathrm{MeV}$ for the baryon chemical potential, if there was equilibrium at the freeze-out of these baryons. Note that the temperature range is roughly in accord with temperature estimates from the transverse momentum distributions, which from large transverse momentum data give $T_{p t} \simeq 230-240 \mathrm{MeV}$ [2]. We should further emphasize that the $p_{T}$ cut of the experiment does not matter here, since in both ratios the masses are the same in numerator and denominator. 
So far we have used two data points to estimate two parameters. To see if these really correspond to the freeze-out parameters of an equilibrium hadron gas, we need further data. These are provided by results for $\Xi^{-} / \Lambda$ and $\bar{\Xi}^{-} / \bar{\Lambda}[2]$. Due to the different masses involved, these ratios have to be corrected for the $p_{T}$ cuts made in the experiment. This reduces the measured ratios, since the $\Xi$-particles have a heavier mass and thus start at higher values of the transverse mass $m_{T}=\left(p_{T}^{2}+m^{2}\right)^{1 / 2}$. We estimate the corresponding correction factor to be

$$
\left[\frac{\Xi^{-}}{\Lambda}\right]_{\text {corr. }}=\left[\frac{\Xi^{-}}{\Lambda}\right]_{\left(m_{T} \text {-cut }\right)} \times \frac{\int_{m_{\Xi}}^{\infty} m_{T} d m_{T} \exp \left(-m_{T} / T\right)}{\int_{m_{\Lambda}}^{\infty} m_{T} d m_{T} \exp \left(-m_{T} / T\right)}
$$

For equal masses, this correction factor is unity; this is the case for the $\bar{\Xi} \Xi \Xi$ and the $\bar{\Lambda} / \Lambda$ ratios. For $\bar{\Xi}^{-} / \bar{\Lambda}$ and $\Xi^{-} / \Lambda$, the correction factor is approximately 0.48 . Using the slopes given by the WA85 collaboration, this leads to $\Xi^{-} / \Lambda=0.10 \pm 0.02$ and $\bar{\Xi}^{-} / \bar{\Lambda}=0.29 \pm 0.10$ for the corrected ratios.

As above, we now calculate the band in the $T-\mu_{B}$ plane corresponding to $\Xi^{-} / \Lambda=$ $0.10 \pm 0.02$; in Fig.11 it is combined with the results shown in Fig.10. We see that this ratio is also compatible with the $T-\mu_{B}$ range obtained in Fig.10. For $\bar{\Xi}^{-} / \bar{\Lambda}=$ $0.29 \pm 0.10$, we include in in Fig.11 only the line corresponding to the value 0.29, without errors, in order to keep the graphics clear. It is seen to be compatible with the region of Fig.10 as well. We therefore conclude that the strange baryon ratios as measured by [2] are quite consistent with equilibrium at freeze-out for $T=200 \pm 20 \mathrm{MeV}$ and

$\mu_{B}=320 \pm 100 \mathrm{MeV}$. Note that this value of $\mu_{B}$ corresponds to $n_{B} \simeq 0.3 \mathrm{fm}^{-3}$, i.e., to about twice nuclear density.

Let us now turn to the ratio $\Lambda / K_{s}^{o}=0.76 \pm 0.16$, measured by [3]. The resulting line is included in Fig.11; it is also absolutely consistent with the previously obtained freeze-out parameters, even though we are now looking at the ratio of a strange baryon to a strange meson.

The picture becomes less consistent, however, if we compare the $\Lambda$ and $\bar{\Lambda}$ to all negative hadrons, i.e., to mainly non-strange mesons. The reported measurements [3] $\Lambda / h^{-}=0.08 \pm 0.01$ and $\bar{\Lambda} / h^{-}=0.015 \pm 0.005$ lead to the cross-over region shown in Fig.12. Although the overlap regions in Figs. 11 and 12 just touch, the region in Fig.12 does point to lower values of the freeze-out temperature as well as baryonic chemical potential. However, before drawing any conclusions, let us consider what the ratios among only mesons indicate.

\subsection{Strange and Non-Strange Mesons}

The ratio $\phi /(\rho+\omega)$ was determined in [5] and [21]; both get a value around $0.3-0.4$ at the highest energy density, and as seen in the dimuon channel. Correcting this by the dimuon branching ratios for $\phi(0.025), \rho$ and $\omega(0.007)$, we get from [21] the value 0.11 \pm 0.01 for the ratio $\phi /(\rho+\omega)$. Since all three mesons are non-strange, the thermal ratio is independent of $\mu_{B}$. In Fig.7 we had shown the behaviour of the thermal $\phi /(\rho+\omega)$ 
as function of $T_{f}$. The measured value is included in the figure; is seen to require very low temperatures, between 100 and $120 \mathrm{MeV}$. It is certainly not compatible with the $T_{f} \simeq 200 \mathrm{MeV}$ found above in the strange baryon analysis.

This conclusion is supported by an analysis of $K / \pi$ ratio. In [3], the ratio $K_{s}^{o} / \pi^{-}$, equivalent to $\left(K^{+}+K^{-}\right) /\left(\pi^{+}+\pi^{-}\right)$, is found to be $0.11 \pm 0.02$ for the integrated data, with a peak value of $0.15 \pm 0.04$ at midrapidity. The resulting bands in the $T_{f}-\mu_{B}$ plane are shown in Fig.13; with values between 100 and $120 \mathrm{MeV}$ (for the integrated data), they also require a considerably lower temperature than found from strange baryons. The same conclusion had been reached in an analysis of the BNL data [4] for the $K^{+} / \pi^{+}$ ratio [22-24], where temperatures around $100 \mathrm{MeV}$ were needed.

\subsection{Conclusions}

We have thus found two sets of data, each in itself compatible with equilibrium hadron ratios, but leading to different freeze-out parameters. Before we comment on possible interpretations, let us note some caveats. We have compared experiments covering different kinematic regions, and we have considered results integrated over kinematic regions showing quite non-uniform behaviour (mid-rapidity vs. forward/backward, for example). This is not really meaningful, and hence everything done here should only serve as an illustration. Ideally, one should use ratios from symmetric nuclear collisions $(A-A)$ at mid-rapidity and integrated over transverse momenta from zero to $1.5-2$ $\mathrm{GeV}$. Hopefully, the $A u$-beam at BNL and the $P b$-beam at CERN will provide such data. - The measurements of vector mesons through dilepton or photon decays may lead to incorrect rate estimates, if the mesons are short-lived enough to decay in the medium [25-28].

If we take the result of our analysis serious in spite of these caveats, they would seem to indicate that the observed strange baryons freeze-out at an earlier and hence "hotter and denser" stage than the mesons. It would be interesting to check if this is also reflected in different temperatures from transverse momentum spectra; there seem to be hints in that direction.

In summary: in this paper we have determined and tabulated the ratios for the various hadron species as function of the freeze-out temperature $T_{f}$ and the baryon number density $n_{B}$, or equivalently, the baryonic chemical potential $\mu_{B}$. These tables should allow a study of the degree and temporal succession of thermalisation for the observed hadron species.

\section{Acknowledgements}

One of the authors (J.C.) thanks O. Villalobos Baillie for comments on the manuscript. The other one (H.S.) gratefully acknowledges stimulating discussions with C. P. Singh. 


\section{References}

1) "Quark Matter '90", Proc. of the VIII'th Int. Conf. on Ultra-Relativistic NucleusNucleus Collisions, Menton, 1990, J.P. Blaizot et al. (editors), Nucl. Phys. A525 (1991).

2) S. Abatzis et al., WA85 Collaboration, Phys. Lett. B244 (1990) 130 and Phys. Lett. B259 (1991) 508.

3) J. Bartke et al., NA35 Collaboration, Z. Phys. C48 (1990) 191

R. Stock et al., NA35 Collaboration Nucl. Phys. A525 (1991) 221c.

4) T. Abbott et al., E802 Collaboration, Phys. Rev. Lett. 64 (1990) 847.

5) C. Baglin etal., NA38 Collaboration, Phys. Lett. B251 (1990) 465 and 472; Phys. Lett. B262 (1991) 362.

6) M. Kataja and P.V. Ruuskanen, Phys. Lett. B243 (1990) 181.

7) S. Gavin and P.V. Ruuskanen, Phys. Lett. B262 (1991) 326.

8) H.J. Crawford, M.S. Desai and G.L. Shaw, Phys. Rev. D45 (1992) 857.

9) C. Greiner, P. Koch and H. Stöcker, Phys. Rev. Lett. 58 (1987) 1825; Phys. Rev. D38 (1988) 2797.

10) "Strange Quark Matter in Physics and Astrophysics", J. Madsen and P. Haensel (editors), Nucl. Phys. B24 (Proc. Suppl.) (1991).

11) J. Rafelski, Phys. Lett. B262 (1991) 333.

12) J. Sollfrank, P. Koch and U. Heinz, Phys. Lett. B253 (1991) 256;

Z. Phys. C52 (1991) 593;

13) J. Cleymans, K. Redlich, H. Satz and E. Suhonen, Z. Phys. C33 (1986) 151.

14) F. Karsch and H. Satz, Phys. Rev. D22 (1980) 480.

15) J. Rafelski, University of Arizona preprint AZPH-TH/91-62

16) H.W. Barz, G. Bertsch, D. Kusnezov and H. Schulz, Phys. Lett. B254 (1991) 332.

17) D. Kusnezov and G.F. Bertsch, Phys. Rev. C40 (1989) 2075.

18) G.E. Brown, J. Stachel and G.M. Welke, Phys. Lett. B253 (1991) 19.

19) J. Schukraft, CERN preprint PPE/91-04 (1991) (unpublished).

20) J. Cleymans, E. Suhonen and G.M. Weber, Z. Phys. C53 (1992) 485.

21) F. Martelli, Talk presented at the Lepton-Photon Symposium, Geneva, Switzerland, July 25 - August 1, 1991.

22) J. Cleymans, H. Satz, E. Suhonen and D.W. von Oertzen, Phys. Lett. B 242 (1990) 111.

23) B. Lukács, J. Zimányi and N.L. Balazs, Phys. Lett. B 183 (1987) 27.

24) P. Koch as reported by U. Heinz in : "Phase Structure of Strongly Interacting Matter." J. Cleymans (ed.), Springer-Verlag, Heidelberg (1990). 
25) P. Koch, U. Heinz and J. Pišut, Phys. Lett. B243 (1990) 149;

Z. Phys. C47 (1990) 477;

26) P. Koch and U. Heinz, Nucl. Phys. A525 (1991) 293c.

27) H.W. Barz, B. Friman, J. Knoll and H. Schulz, Phys. Lett. B254 (1991) 315.

28) P-Z. Bi and J. Rafelski, Phys. Lett. B262 (1991) 485.

29) J. Cleymans, K. Redlich, H. Satz and E. Suhonen, Z. Phys. C33 (1986) 151.

30) N.J. Davidson, H.G. Miller, R.M. Quick and J. Cleymans, Phys. Lett. B255 (1991) 105; D.W. von Oertzen et al. Phys. Lett. B274 (1992) 128.

31) P. Koch, B. Müller and J. Rafelski, Phys. Rep. 148 (1986) 167. 


\section{Figure Captions}

Fig. 1: Strange chemical potential $\mu_{S}$ as a function of baryon chemical potential $\mu_{B}$ for temperature values from $T=150 \mathrm{MeV}$ to $T=300 \mathrm{MeV}$. The solid line was calculated for a temperature of $10 \mathrm{GeV}$.

Fig. 2.: Baryon density $n_{B}$ as function of baryon chemical potential $\mu_{B}$ at temperature $T=200 \mathrm{MeV}$ for pointlike baryons (dash-dotted line) and for baryons with hardcore repulsion (full line).

Fig. 3: Ratio of all pions to thermal pions as function of temperature $T$ for baryon chemical potential $\mu_{B}=200 \mathrm{MeV}$. The full line includes the contributions from the fundamental hadron multiplets only, the dash-dotted line contributions from all resonances with masses up to $2 \mathrm{GeV}$.

Fig. 4: Ratio of all pions to thermal pions as function of baryon chemical potential $\mu_{B}$ for temperatures $T=170 \mathrm{MeV}$ (full line) and $T=200 \mathrm{MeV}$ (dash-dotted line); calculations include the fundamental hadron multiplets only.

Fig. 5: Ratio of all kaons to thermal kaons as function of temperature $T$ for baryon chemical potential $\mu_{B}=200 \mathrm{MeV}$; calculations include the fundamental hadron multiplets only.

Fig. 6: Ratio of all kaons to thermal kaons as function of baryon chemical potential $\mu_{B}$ for temperatures $T=170 \mathrm{MeV}$ (full line) and $T=200 \mathrm{MeV}$ (dash-dotted line). Calculations include the fundamental hadron multiplets only.

Fig. 7: Ratio of $\phi$ to $\rho+\omega$ as function of temperature $T$; calculations include all resonances up to masses of $2 \mathrm{GeV}$.

Fig. 8: Ratio of $\phi$ to $\rho+\omega$ as function of baryon chemical potential $\mu_{B}$ at temperature $T=200 \mathrm{MeV}$, including contributions from all resonances with masses up to 2 $\mathrm{GeV}$.

Fig. 9: Ratio of all $\Xi$ 's to thermal $\Xi$ 's as function of temperature $T$ for baryon chemical potential $\mu_{B}=200 \mathrm{MeV}$; calculations include the fundamental hadron multiplets only.

Fig. 10: $T-\mu_{B}$ region consistent with the measured ratios for $\bar{\Lambda} / \Lambda$ and $\bar{\Xi} / \Xi$; the experimental error limits of the ratios are indicated.

Fig. 11: $T-\mu_{B}$ region consistent with the measured ratios for $\bar{\Lambda} / \Lambda, \bar{\Xi} / \Xi, \Xi^{-} / \Lambda, \bar{\Xi}^{-} / \bar{\Lambda}$, and $\Lambda / K_{s}^{o}$; the experimental error limits of the ratios are indicated, except for the last two ratios.

Fig. 12: $T-\mu_{B}$ region consistent with the measured ratios for $\Lambda / h^{+}$(dashed line) and for $\bar{\Lambda} / h^{-}$ratio (full line); the experimental error limits of the ratios are indicated.

Fig. 13: $T-\mu_{B}$ region consistent with the measured ratio for $K_{S}^{0} / h^{-}$; the experimental error limits of the ratio are indicated. 